Revue d'histoire de l'Amérique française

REVUE D.HISTOIRE DE L'AMÉRIQUE FRANÇAISE

\title{
Origine du nom de la ville de Montréal
}

\section{Jean Poirier}

Volume 46, numéro 1, été 1992

Montréal 1642-1992

URI : https://id.erudit.org/iderudit/305046ar

DOI : https://doi.org/10.7202/305046ar

Aller au sommaire du numéro

Éditeur(s)

Institut d'histoire de l'Amérique française

ISSN

0035-2357 (imprimé)

1492-1383 (numérique)

Découvrir la revue

Citer cet article

Poirier, J. (1992). Origine du nom de la ville de Montréal. Revue d'histoire de l'Amérique française, 46(1), 37-44. https://doi.org/10.7202/305046ar
Résumé de l'article

Des hypothèses sur l'origine du nom de Montréal, la toponymie retient celle, plus près de la réalité, qui y voit une variante de mont Royal. d'utilisation que vous pouvez consulter en ligne.

https://apropos.erudit.org/fr/usagers/politique-dutilisation/ 


\title{
ORIGINE DU NOM DE LA VILLE DE MONTRÉAL ${ }^{1}$
}

\author{
JEAN POIRIER \\ Adjoint au président \\ Commission de toponymie du Québec
}

\section{RÉSUMÉ}

Des hypothèses sur l'origine du nom de Montréal, la toponymie retient celle, plus près de la réalité, qui y voit une variante de mont Royal.

\section{ABSTRACT}

Among the hypotheses concerning the origin of Montréal's name, the most acceptable to Toponymy is the one that finds it to be a variant of mont Royal.

Quelques hypothèses ont été formulées sur l'origine du toponyme Montréal. Plusieurs voient dans la forme Montréal une variante du toponyme mont Royal, nom donné par Jacques Cartier en 1535 à la colline qui domine la ville. D'autres, en revanche, prétendent que le nom Montréal rappelle le nom ou le titre d'un personnage. L'historien Marcel Trudel posait la question suivante en 1963: «D'où vient ce toponyme mont Royal? en l'honneur du cardinal de Médicis, archevêque de Monreale? en l'honneur de Claude de Pontbriand, fils du seigneur de Montréal? ou tout simplement en l'honneur du roi? aucune explication sûre n'a encore été trouvée ${ }^{2} . »$

Un examen détaillé de documents anciens, appuyé par les méthodes d'analyse de la toponymie d'une part et par celle de la linguistique d'autre part, accrédite l'idée que le toponyme Montréal est une variante du toponyme primitif mont Royal.

Pour connaître l'origine et la signification d'un nom de lieu, le principe de base, en toponymie, est de se référer obligatoirement aux

1 Communication présentée au XVIII congrès annuel de la Société d'histoire coloniale française - The French Colonial Historical Society tenu à Montréal du 21 au 23 mai 1992.

2 Marcel Trudel, Histoire de la Nouvelle-France, I, Les vaines tentatives, 1524-1603 (Montréal, Fides, 1962), 98, note 9. 
formes les plus anciennes attestées dans les documents. Albert Dauzat, le célèbre toponymiste français, résume ainsi ce principe de la toponymie: «Il serait imprudent d'aborder la recherche étymologique d'un nom de lieu en tablant uniquement sur la forme actuelle. Il faut remonter dans le passé et renouer patiemment la chaîne des formes qui l'ont précédée jusqu'à la plus ancienne. Procéder autrement serait s'exposer aux bévues et erreurs les plus graves ${ }^{3}$.» Pour suivre cette approche scientifique, nous avons dressé la chronologie des formes anciennes du toponyme Montréal, de 1535 à 1754, chronologie qui paraît en annexe. Il convient donc d'examiner les attributions primitives de ce nom de lieu, lesquelles sont déterminantes pour connaître l'origine de ce toponyme. Ce qui ressort de cet examen, c'est que, d'une part, Cartier a donné le nom de mont Royal à une colline et que, d'autre part, le toponyme Montréal n'est pas attesté dans ses relations de voyages en Nouvelle-France.

Jacques Cartier fournit l'attestation la plus ancienne du nom de lieu mont Royal quand il écrit dans sa Relation de 1535-1536: «Et au parmy d'icelles champaignes, est scituée et assise ladicte ville de Hochelaga, près et joignant une montaigne, qui est, à l'entour d'icelle, labourée et fort fertille, de dessus laquelle on voyt fort loing. Nous nommasmes icelle montaigne le mont Royal ${ }^{4} . »$

Ce n'est que quarante ans plus tard, soit en 1575, qu'il est de nouveau question de ce nom de lieu dans les documents français consultés. Dans sa Cosmographie universelle..., André Thevet, historiographe du roi de France, écrit en 1575: «Ce fut en leur terre que les Francoys bastirent, il y a longtemps, un fort, pres une montaigne qu'ils nommerent Mont-royal ${ }^{5}$.» Dans ce texte, André Thevet, qui indique le nom de lieu primitif donné par Jacques Cartier, commet une erreur cependant quand il affirme que les Français ont construit un fort à cet endroit. Le fort en question ou plus précisément les deux forts avaient été érigés par Cartier en 1541 à Charlesbourg Royal, aujourd'hui Cap-Rouge, à quelque distance en amont du site actuel de Québec.

C'est dans La Cosmographie universelle de tout le monde..., ouvrage également produit en 1575 par François de Belleforest, un autre historiographe français, que le nom de lieu Montréal est attesté

3 Albert Dauzat, Les noms de lieu. Origine et évolution (Paris, Delagrave, 1957), 4.

4 H. P. Biggar, Voyages of Jacques Cartier (Ottawa, F. A. Acland, «Publications of the Public Archives of Canada», $\left.\mathrm{n}^{\circ} 11,1924\right), 154-155$.

5 André Thevet, La Cosmographie universelle illustrée de diverses figures des choses des plus remarquables veües par l'auteur, \& incogneües de noz anciens et modernes (Paris, 1575, 2 vol.), fo 1010. 
pour la première fois. En effet, cet auteur écrit: «laquelle ville les Chrestiens appellerent Montreal ${ }^{6} »$. Ce qui frappe dans ce texte, c'est que le toponyme s'applique au village indien que Cartier avait identifié, en 1535, sous le nom de "ville de Hochelaga», et que celleci porte pour Belleforest la dénomination de Montreal'. C'est la forme Montréal qui devait par la suite s'imposer au lieu de mont Royal pour désigner l'île et la ville.

Comment expliquer le nom de lieu Montreal pour désigner en 1575 un lieu habité à proximité de la colline qui avait été nommée en 1535 mont Royal? Deux raisons principales permettent de répondre à cette question. La première relève de la linguistique diachronique; la seconde concerne l'influence qu'a eue la traduction italienne des Relations de Cartier chez François de Belleforest. Il convient d'examiner d'abord la première raison. Walther von Wartburg, un des grands romanistes qui ont fait la synthèse de la linguistique historique, démontre clairement qu'au $\mathrm{XVI}^{\mathrm{e}}$ siècle les formes royal et real, qui avaient le même sens, étaient toutes deux en usage en France. En effet, la forme real est attestée en français du $\mathrm{IX}^{\mathrm{e}}$ au XVI $\mathrm{XV}^{\mathrm{e}}$ siècle, tandis que la forme royal l'est depuis le XIII ${ }^{e}$ siècle $^{8}$. Cette réalité linguistique permet de comprendre pourquoi les auteurs de cette époque ont tantôt écrit real, tantôt royal. Cet emploi de real pour royal dans Montréal n'est pas un cas unique dans la toponymie de la Nouvelle-France au $\mathrm{XVI}^{\mathrm{e}}$ siècle. Voici un autre exemple également extrait de la toponymie de Jacques Cartier qui illustre ce fait linguistique. Lors de son premier voyage en Nouvelle-France, en 1534, Cartier avait nommé cap Royal une saillie de terre de l'île de Terre-Neuve. Aujourd'hui, cette élévation qui s'avance dans la mer porte le nom de Bear Head ${ }^{9}$. Or n'est-il pas étonnant de constater que, sur la mappemonde datée de 1546 du cartographe français Pierre Desceliers, savant originaire de Dieppe, ce nom de lieu est écrit $C$. Real et non plus $C$. Royal comme l'avait baptisé Jacques Cartier douze ans auparavant ${ }^{10}$ ? Cet exemple prouve encore une fois que les auteurs, selon leur usage linguistique, pouvaient écrire indifféremment real ou royal au XVI ${ }^{\mathrm{e}}$ siècle. Comme on le voit, le nom de lieu Montreal indiqué par Belleforest est une autre forme de mont Royal.

6 François de Belleforest, La Cosmographie universelle de tout le monde... (Paris, 1575, 2 vol.), II: 2191.

7 Biggar, op. cit., 155.

8 Walther von Wartburg, Französisches Etymologisches Wörterbuch (Basel, R. G. Zbinden, 1962), X: 201.

9 Christian Morissonneau, Le langage géographique de Cartier et de Champlain (Québec, Presses de l'Université Laval, coll. «Choronoma 7», 1978), 29.

10 Biggar, op. cit., 192. 
La deuxième raison qui explique la forme Montreal chez Belleforest, en 1575, est l'influence qu'a exercée, chez cet auteur, la traduction italienne des relations de voyages de Jacques Cartier; l'auteur s'étant inspiré de cette traduction plutôt que du Brief récit... de Cartier, publié en France en 1545 et qui est la narration du voyage de $1535-1536^{11}$. Cette source écrite dont s'est inspiré cet historiographe français pour rédiger notamment sa Cosmographie..., en 1575 , est le troisième volume des Navigationi et viaggi... publié en 1556 - et dont la première réimpression est de 1565 - par le géographe vénitien Giovanni Battista Ramusio. Ce livre a connu un succès considérable, attesté par de nombreuses réimpressions. C'est principalement dans la version de Ramusio que l'Europe lettrée allait être informée des entreprises de Jacques Cartier ${ }^{12}$. Dans sa Cosmographie de 1575, François de Belleforest lui-même rend fréquemment hommage à Ramusio et, tout en le condensant, suit de près, pour Cartier en particulier, les récits qu'il lui emprunte ${ }^{13}$. Nous reproduisons le plan de Ramusio paru dans la première réimpression, en 1565, des ses Navigationi... Ce plan intitulé La terra de Hochelaga nella Nova Francia indique Monte Real, toponyme bien à sa place sur la colline ${ }^{14}$. Donc, de l'aveu même de François de Belleforest, qui insiste sur l'importance du rôle de Ramusio dans ses écrits, on peut comprendre qu'il ait inscrit Montreal au lieu de mont Royal, la première forme étant plus proche de Monte Real indiquée par le géographe vénitien. Plus surprenant cependant est le fait que sur ce plan de La terra de Hochelaga, qui est l'œuvre du cartographe vénitien Giacomo Gastaldi, ce ne sont pas les formes italiennes Reale ou Regale qui apparaissent mais la forme Real. Bien que la raison de l'emploi, chez Gastaldi, de Real au lieu de l'italien Reale nous soit inconnue, il est vraisemblable de penser qu'il a pu s'inspirer d'un document espagnol ou portugais.

Le passage du nom de lieu mont Royal à Montreal, en 1575, est donc clairement démontré par les textes anciens, la linguistique, la cartographie et les récits de voyages de Ramusio. En outre, l'emploi sous la plume de Pierre Boucher en 1664 de mont-Royal au lieu de Montréal pour désigner l'île montre le lien évident qui existe entre ces

11 Michel Bideaux, Jacques Cartier. Relations (Montréal, Presses de l'Université de Montréal, 1986), 34.

12 Ibid., 38.

13 Ibid., 286, n. 221.

14 Giovanni Battista Ramusio, Terzo volume delle navigationi et viaggi nel quale si contengono le navigationi al Mundo Nuovo... (Venise, Giunti, 1556 [réédité en 1565, 1606, 1613 et à Turin en 1978]). C'est dans la réédition de 1565 que paraît Monte Real sur le plan de Ramusio reproduit ici. 


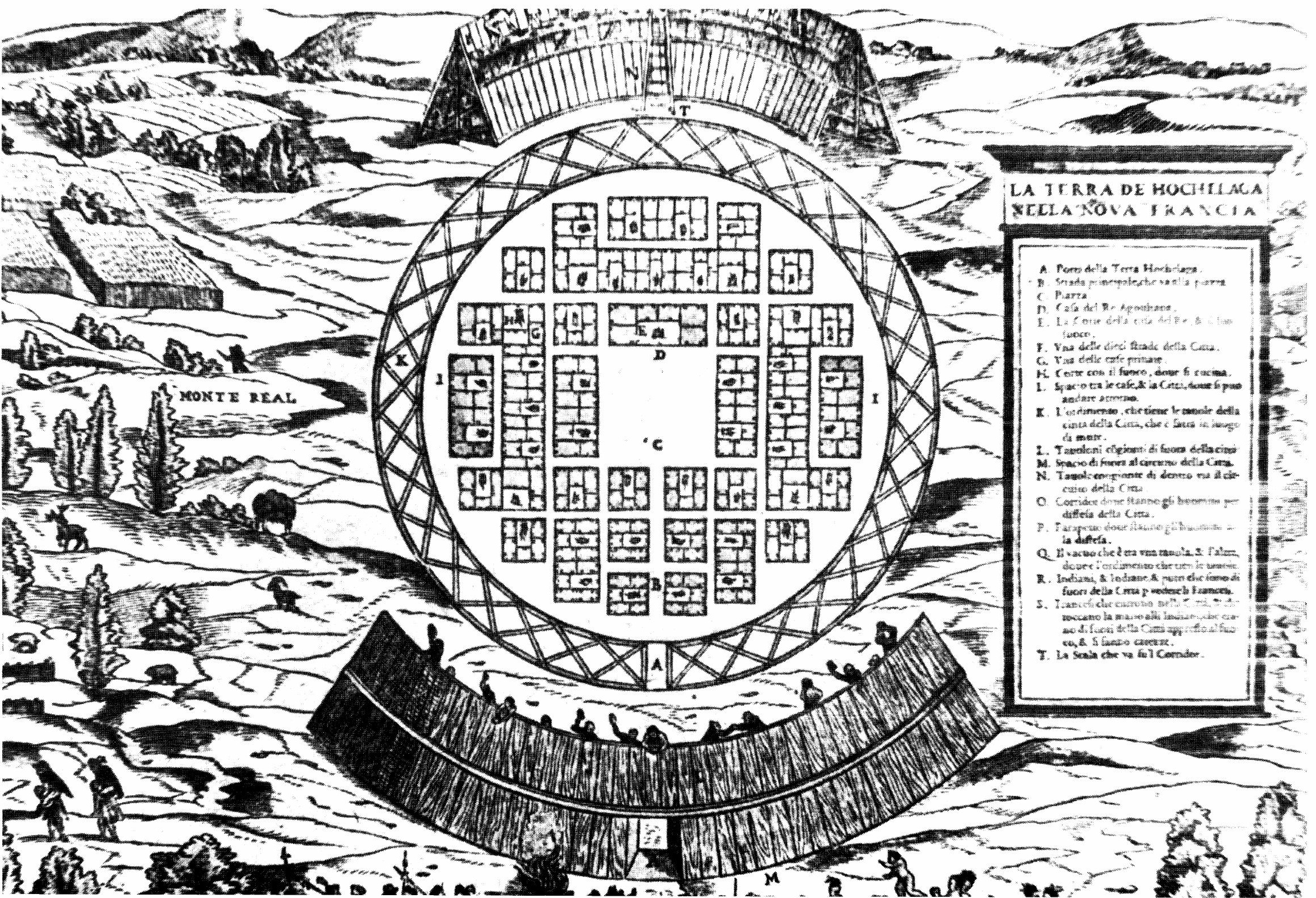


deux formes: «Mont-Royal, écrit ce gouverneur de Trois-Rivières, qui est la dernière de nos habitations [...] est située dans une belle grande Isle nommée l'Isle du mo[n]t-Royal ${ }^{15}{ }$.

On a prétendu, et on prétend encore parfois, que le toponyme Montréal tire son origine d'un personnage, d'un titre, voire même d'une seigneurie. Certains textes peuvent inciter à souscrire à cette théorie. Voici la première hypothèse qui se résume ainsi. Le cardinal Jean Le Veneur, grand aumônier de France, appuyé par le cardinal Hippolyte de Médicis, neveu de Clément VII, pape de 1523 à 1534, a obtenu une déclaration du Saint-Père selon laquelle la bulle pontificale partageant les continents nouveaux entre les couronnes d'Espagne et du Portugal ne concernait que les terres connues et non celles ultérieurement découvertes par les autres couronnes. C'est pour remercier le cardinal de Médicis, archevêque de Monreale, en Sicile, que François Ir $^{\text {r }}$ aurait prescrit à Jacques Cartier de donner le nom de Montreal à un lieu sur les terres qu'il découvrirait ${ }^{16}$.

Une autre théorie soutenue par quelques auteurs, dont notamment au XIX ${ }^{\mathrm{e}}$ siècle l'historien Gerald E. Hart, prétendent que Montréal tire son appellation du titre d'un compagnon de Jacques Cartier ${ }^{17}$. Dans sa Relation de 1535-1536, le «découvreur» du Canada écrit que, pour aller à Hochelaga, il était «acompaigné de partie des gentilzhommes, savoir: de Claude du Pontbryand, eschansson de monseigneur le Daulphain ${ }^{18} »$. Ce gentilhomme était Claude de Pontbriant dit Montréal ${ }^{19}$.

Rien cependant ne permet d'affirmer - et tout indique le contraire - que Montréal ait ces origines. Le nom de lieu Montréal ne paraît nulle part dans les relations de Jacques Cartier et de Roberval, ni dans les documents postérieurs comme les écrits de Jean Fonteneau dit Jean Alfonse, notamment dans sa Cosmographie (1544) et dans ses Voyages Avantureux (1559). Montréal ne paraît pas non plus sur les cartes, mappemondes et planisphères qui ont été dressés à la suite des voyages de Cartier tels ceux des cartographes Desliens (1541), Cabot (1544), Desceliers (1546 et 1550), Mercator (1569). De plus, lorsque Jacques Cartier a honoré des personnages dans la toponymie de la

15 Pierre Boucher, Histoire véritable et naturelle des mours et productions du pays de la Nouvelle-France, vulgairement dit le Canada (Paris, Florentin Lambert, 1664), 22.

16 Jean-François Hénault, «Extrait de la généalogie de la Maison Le Veneur, comtes de Tillières de Carrouges», Nova Francia (Paris), VI (1931): 340-343.

17 Pierre-Georges Roy, Les noms géographiques de la province de Québec (Québec, Cie de publication Le Soleil, 1906), 282.

18 Biggar, op. cit., 148.

19 Robert Prévost, Montréal de France (Montréal, Libre Expression, 1991), 121-122. 
Nouvelle-France, il l'a fait d'une manière exacte et sans ambiguïté. Il en fut ainsi, par exemple, pour l'île Brion, qui fait partie de l'archipel des îles-de-la-Madeleine dans le golfe du Saint-Laurent. En la baptisant de ce nom en 1534, Cartier a voulu honorer Philippe Chabot, seigneur de Brion, amiral de France et de Bretagne. C'est ce personnage qui établira le 30 octobre 1534 la commission relative au deuxième voyage de Cartier $^{20}$.

Signalons à titre de renseignement que le toponyme Montréal est assez répandu en France, en Espagne, ainsi qu'en Italie. Dans ce dernier pays, il est attesté sous la forme Monreale. Le toponymiste français Ernest Nègre démontre que les quelque vingt noms de lieux Montréal de France sont des formations dialectales attestées dès les $\mathrm{XII}^{\mathrm{e}}$ et XIII ${ }^{\mathrm{e}}$ siècles sous les formes suivantes: Mons Regalis en 1145; Monte Regali en 1210; Mons Real en 1231; apud Montem Regualem en 1258 et Monteregali en 1272. Ils signifient selon cet auteur «dépendant directement du roi ${ }^{21}$ ». Ajoutons aussi que le transfert en Nouvelle-France d'un Montréal de France n'a jamais été démontré et ne peut pas l'être quand on connaît l'origine de Montréal au Canada.

Le constat s'impose: les documents anciens attestent que le nom de lieu mont Royal, toponyme stable et sans concurrent, a été nommé ainsi par Jacques Cartier en 1535. Ce nom a-t-il été donné en l'honneur précisément du roi de France ou a-t-il le sens général de «qui est digne d'un roi»? Cette question restera sans réponse puisque Cartier n'a pas donné d'explication. La documentation historique montre également que la forme Montréal, qui est apparue quarante ans plus tard, soit en 1575, est une variante de mont Royal.

Quand l'explorateur français Samuel de Champlain, sur sa carte de 1612 intitulée Carte géographique de la Nouvelle-France a indiqué Montreal pour désigner la colline que Jacques Cartier avait baptisée mont Royal, il reprenait la forme qu'avait écrite François de Belleforest en 1575. Cependant, dans son livre de 1613 intitulé Les Voyages du Sieur de Champlain Xaintongeais ainsi que dans celui de 1632 Les voyages de la Nouvelle-France occidentale dite Canada, ce géographe revient au nom de lieu primitif mont Royal pour identifier cette colline $^{22}$. Cela signifie que le nom de lieu Montréal devait être bien vivant pour que Champlain désigne ce mont sous cette appellation en 1612. Comme des auteurs, en particulier Champlain, remplacent

20 Bideaux, op. cit., 323, note 157.

21 Ernest Nègre, Toponymie générale de la France (Genève, Droz, 1991, 3 vol.), II: 1159-1170.

22 Samuel de Champlain, «Les voyages du sieur de Champlain», Euvres de Champlain (Montréal, Éd. du Jour, 1973), 1613: 391; 1632: 839. 
Montréal par le nom primitif mont Royal, on est en présence de deux toponymes parallèles pour désigner la colline. Finalement, la colline conservera son nom originaire, soit mont Royal, tandis que l'appellation Montréal servira à dénommer l'île où est située cette colline et plus tard la ville.

En effet, l'île où est bâtie la ville de Montréal a reçu son nom dans la première moitié du XVII ${ }^{e}$ siècle. Dans la table pour connaître les lieux remarquables de sa carte de 1632, Samuel de Champlain note à deux reprises que ce territoire s'appelle Isle de Mont-real ${ }^{3}$.

23 Champlain, op. cit., 1632, III: 1385-1389. Autres publications consultées pour la présente étude: René Bouchard et al., Itinéraire toponymique du chemin du Roy QuébecMontréal (Québec, Commission de toponymie, «Études et recherches toponymiques», 2, 1981). Commission de toponymie, Itinéraire toponymique du Saint-Laurent. Ses rives et ses îles (Québec, «Études et recherches toponymiques», 9, 1984). W. F. Ganong, Crucial Maps in the Early Cartography and Place-Nomenclature of the Atlantic Coast of Canada (Toronto, University of Toronto Press, 1964). Richard Hakluyt, The Third and Last Volume of the Voyages, Navigations, Traffiques and Discoveries of the English Nation... (London, 1600). Ch.-A. Julien et Th. Beauchesne, Les Français en Amérique pendant la premiere moitié du XVI siecle (Paris, 1946). Hormisdas Magnan, Dictionnaire historique et géographique des paroisses, missions et municipalités de la province de Québec (Arthabaska, Imprimerie d'Arthabaska, 1925). Jean Poirier, "_le de Montréal», Canoma (Énergie, Mines et Ressources Canada), 5,2 (décembre 1979). «Montréal», Le Toponyme (Bulletin d'information sur les noms de lieux, Québec, Commission de toponymie), 3,3 (novembre 1985). «Le nom de lieu Montréal au Québec», Nouvelle Revue d'Onomastique (Paris), 7-8 (1986). Roger Schlesinger et Arthur Stabler, André Thevet's North America: A Sixteenth Century View (Montréal, McGill-Queen's University Press, 1986). 